\title{
Los Andes del Sur
}

\section{Cordillera \\ Con tus siete pueblos camin \\ en tus faldas acigïeñadas caminas la noche y el día..

Tras más de un recorrido por la cordillera, el autor reflexiona sobre la relación del pueblo chileno con los Andes. Mediante preguntas y conclusiones se reivindica lo que ha sido bistóricamente una barrera despoblada, agreste y abandonada, a la cual se le da la espalda, más que un territorio con el potencial de dar cabida a todo un imaginario.

After walking several routes along and across the mountain range, the author reflects about the relation between Chilean people and their most important geographic reference, the Andes. What has been an empty, tough and abandoned barrier, and has also been given the back by the dwellers of the Chilean territory, he vindicates it as a location that would give place to a collective consciousness.

De los 7 países sudamericanos que comparten la cordillera de los Andes seis caminan con ella; Chile se bajó.

Hace muchos años que camino por la cordillera y cada vez la veo más abandonada.

Veo con tristeza cómo se va despoblando.

Mientras escribo miro por la ventana las siete cumbres que me rodean: el cerro Damas, el San Ramón, Bandurrias, Coironal, Purgatorio, Los Azules y Pechuga de Tórtola, y constato esta enorme belleza perdida para la mayoría de los chilenos.

Comparo este abandono con la cordillera de los otros países sudamericanos en los que he trabajado como escultor en tiempos prolongados: Quito en Ecuador, Alajuela en Costa Rica y Cochabamba en Bolivia.

Sewell es una luz que se apaga lentamente en la cordillera de Chile.

Es como si existiera una fuerza chamánica oscura que empuja a los chilenos hacia los valles.

En esta lucha también se han empeñado ingenieros, políticos y especialmente arquitectos, la mayoría de ellos desde Santiago. En la práctica han terminado sumándose a los grandes proyectos inmobiliarios y urbanísticos, intentando solucionar el problema en la parte de adentro de la ciudad.

Esta concentración de los chilenos en los 90 km cuadrados de la ciudad de Santiago no sólo tiene como consecuencia una depresión colectiva, sino el hecho que la cordillera se ha ido convirtiendo poco a poco en un sitio eriazo.

La tarea que deja Gabriela Mistral en sus tres versos: subir a los Andes de nuevo a su séptimo pueblo, es también la tarea de este escrito.

Nos juntamos en un colectivo ${ }^{1}$ cuya empresa mayor fue el "Paso de los Andes" entre febrero y marzo de 2001.

Repetimos el Cruce de los Andes, una empresa que por su sola fuerza se constituye en un hito cultural: hace 200 años 4.000 hombres, 10.600 mulas y 1.200 caballos pasaron por una pequeña huella de $30 \mathrm{~cm}$ de ancho y 370 kilómetros de largo a 4.700 metros de altura, entre el Mercedario y el Aconcagua, las cumbres más altas de la cordillera de los Andes, para fundar la modernidad del cono sur de América.

Por esa pequeña huella entró la ilustración con todos sus beneficios: la ciencia, la democracia y la república.

Agregamos al cruce un largo viaje de exploración de la cordillera del tramo entre el Choapa y el Bío Bío, a una altura promedio que va entre los 5.000 y los $2.000 \mathrm{~m}$.

Este viaje de aproximadamente $1.200 \mathrm{~km}$ por tierra, que probablemente ningún chileno había hecho antes, comenzó en 1997.

Nuestro objetivo, llegar al año 2010 habiendo trazado un camino a través del territorio andino de Chile, recogiendo la mayor cantidad de información, mirando los Andes desde las distintas áreas de la cultura y un mapa completo de este camino de los Andes del sur; siguiendo las huellas, casi perdidas, del ingeniero Luis Risopatrón, que hace 100 años puso los hitos de acero en los límites de la cordillera, incorporando, técnicamente, los Andes al territorio de Chile.

Muchas de las preguntas y conclusiones de este escrito son el resultado del viaje de reconocimiento; al volver tomamos conciencia que no se ha construido el Tihuanacu de los Andes del sur, ni siquiera el Quito ni el Potosí.

No se ha escrito la novela de los Andes.

No se ha escrito la música de los Andes del sur.

No hemos podido poner una escultura en la cordillera.

Esta demarcación cultural del territorio andino de Chile es una tarea dura, que ni los organismos de cultura del país y ni los empresarios están dispuestos a financiar.

La apropiación cultural de los Andes aparece como una tarea insólita en un momento en que todo el "sistema de las artes" está dirigido a producir artistas para Nueva York.

Los poetas sí.

Gabriela Mistral comenzó a escribir en 1910 en el mismo momento que Risopatrón instalaba los hitos limítrofes.

En mi gremio, los escultores, nos sumamos a principios del siglo con el indudable andinismo de la generación de Samuel Román y Marta Colvin. Pero la mayor parte de las veces, los Andes en Chile son un desierto abandonado a mineros de fortuna que cortan sus caminos; o buscadores de energía eléctrica que meten los ríos en tubos de concreto.

No es el caso de nuestros hermanos del altiplano perú-boliviano que siempre han vivido, sabiamente, en los Andes entre 1.000 y 5.000 metros.
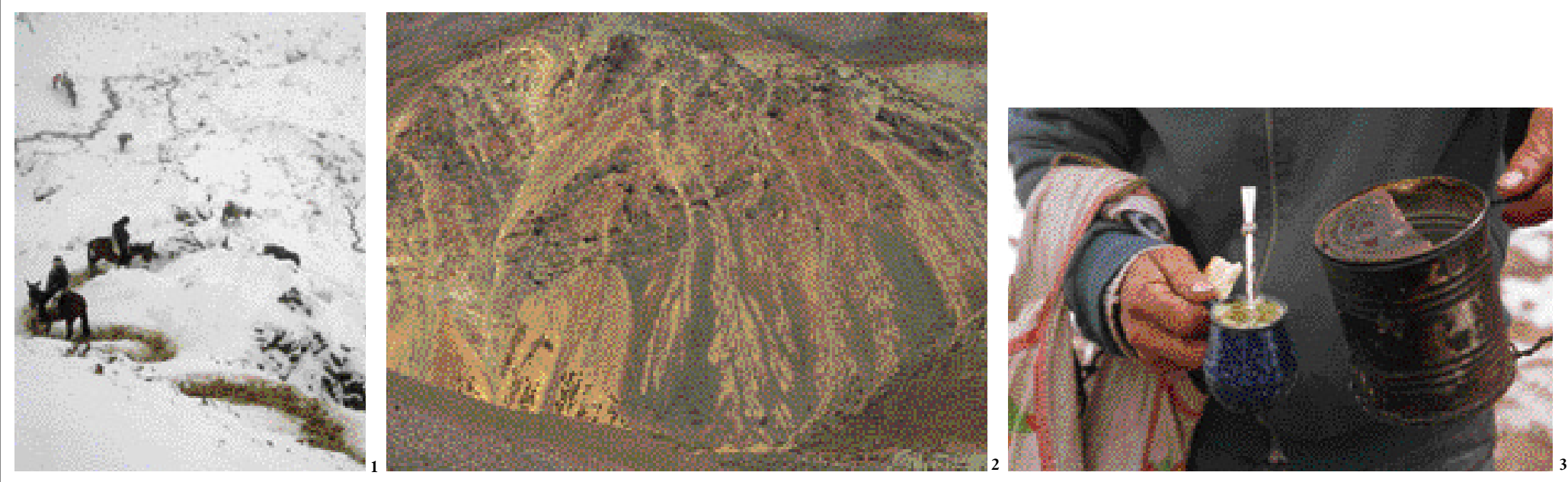


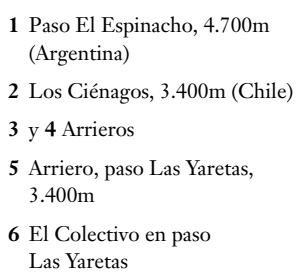

Ellos colonizaron nuestra cordillera, nos enseñaron a vivir en ella, trazaron nuestras primeras huellas y canales de regadío. "Mi imperio llega donde la llama llega", dijo el Inca una vez, para describir la andinidad de su cultura (Evelio Echevarría).

La guerra del Pacífico, si bien amplió nuestro territorio marítimo, cortó relaciones con nuestros hermanos mayores de los Andes. Ha sido tan grande la desconsideración con esa cultura, que eliminamos la mayor ofrenda simbólica de los Incas al valle de Santiago, trasladando al niño-momia del cerro El Plomo a un refrigerador para exhibirlo para nuestro deshonor en el museo de la Quinta Normal.

\section{¿Es Chile un país andino?}

Los Andes de Chile son la mitad del territorio del país y están vacíos.

El vacío cultural de los Andes es el vacío de Chile. Los Andes de Chile están formados por 15.000.000 de cerros, un cerro para cada chileno.

¿Quién se hace cargo de su montaña?

Suiza entera cabe en el tramo de los Andes desde el Choapa hasta el Maule.

Durante el verano pasado me encontré con la excelente novela del arquitecto Oscar Bustamante, llamada "Explicación de todos mis errores", donde se cuenta la historia de un chileno contemporáneo que dos veces intenta vivir en los Andes.

Carlos Overnead sube a los 21 años a las cordilleras del Maule y decide pasar un año entero allí "para templarse como guerrero."

Carlos se queda sólo en la Laguna del Dial a 2.500 metros. En los nacimientos del río Melado se quedó "para que esa naturaleza dura marcara en mí huellas indelebles".

Pasa el tiempo y Carlos comienza a ver la primera cara del demonio de las alturas ...
"La soledad que impregna, incluso al viento, a los pastos y a la lluvia, los árboles la sienten, y entrecruzando sus ramas al viento se cobijan de su violencia".

El autor no cuenta cómo llenaba Carlos su día ni sus rutinas de mulas y cinchones, ni cómo dormía en su casa de piedra, ni qué soñaba sobre los pellones de oveja, ni la dura recopilación diaria de la poca leña del lugar, sólo nos cuenta del primer golpe de soledad violenta de los Andes. Esa soledad que no existe en el resto de los Andes del Norte, ni la he visto en ninguna otra montaña, ni en los Alpes, ni en las Black Mountains de Gales, ni en los montes Líbanos. En ellas nunca se está solo, cada lugar está marcado por miles de vidas que desde muy antiguo fueron dejando su presencia en signos culturales.

En la tarea de tiempo completo de solamente estar ahí, se va dibujando la silueta del segundo demonio de nuestra cordillera. La primera trabaja a Carlos Overnead desde dentro hacia fuera por el simple hecho de no ser piedra.... "qué extraño estar desterrado en este anfiteatro sideral de piedras, aguas, volcanes y luz y al mismo tiempo querer ser parte de todo esto. Ser piedra, ser pasto y agua, $y$ hay momentos en que me revuelco en ellos, en que sumido en las arenas siento que pronto voy a convertirme en una porción infinitesimal del universo...". "Devorando nieve voy a ser hielo, y no, no me convierto en piedra, no me convierto en viento desnudo sobre un farellón...no soy materia primigenia. Sólo soy alma, sólo soy conciencia”. Otra pata del alicate que aprieta el diablo de la hondonada de los Andes está al final del libro: "Si amanece todos los días y nacemos y morimos sin que el pasto se agite".

Bustamante señala, más adelante, un tercer diablo de las alturas:
Nuestra manera de pensar la cordillera y sus habitantes, los arrieros.

El arriero podría ser un buen sujeto para desmitificar esta sensibilidad inútil.

Después de haber vivido la vida entera entre canteros y arrieros, comienzo a comprender su verdad de hombres simples, igual que cualquier otro. La única manera de llegar a entenderlos es con una sensibilidad vinculada a nuestras raíces mediterráneas, que tan bien trabajaron Cervantes, Quevedo y Machado. Que reaparecen en Gabriela Mistral.

La mitad del territorio de Chile está vacía.

El vacío de los Andes es el vacío de Chile.

A veces pienso que simplemente no existe ese vacío para la mayoría de los chilenos que están de acuerdo, secretamente, en que los Andes deben seguir así, como una gran muralla detrás de la cual es mejor no saber lo que pasa.

Poco sirve la estética romántica y todos sus derivados.

Tampoco sirven las estéticas realistas: el realismo socialista, el realismo mágico, el surrealismo, han sido inútiles para descifrar las aciones de los Andes. Mucho menos ha servido el estructuralismo porque trabaja con el nombre de las cosas, un conceptual se perdería en la cordillera porque en los Andes todo se llama igual. Lo dijo Luis Risopatrón hace 100 años en su libro Cordillera de los Andes: "Los arrieros se esfuerzan por darles a los accidentes geográficos un nombre que sea fácil recordar. Así, entre los ríos se repiten hasta el cansancio los mismos nombres de colorado, verde, claro, salinillas, y entre los cajones y quebradas: yeguas muertas, caballo muerto, caballo quebrado, caballo perdido, potrerillo de los caballos, potrero escondido, etc.

Me impresiona también la falta de información
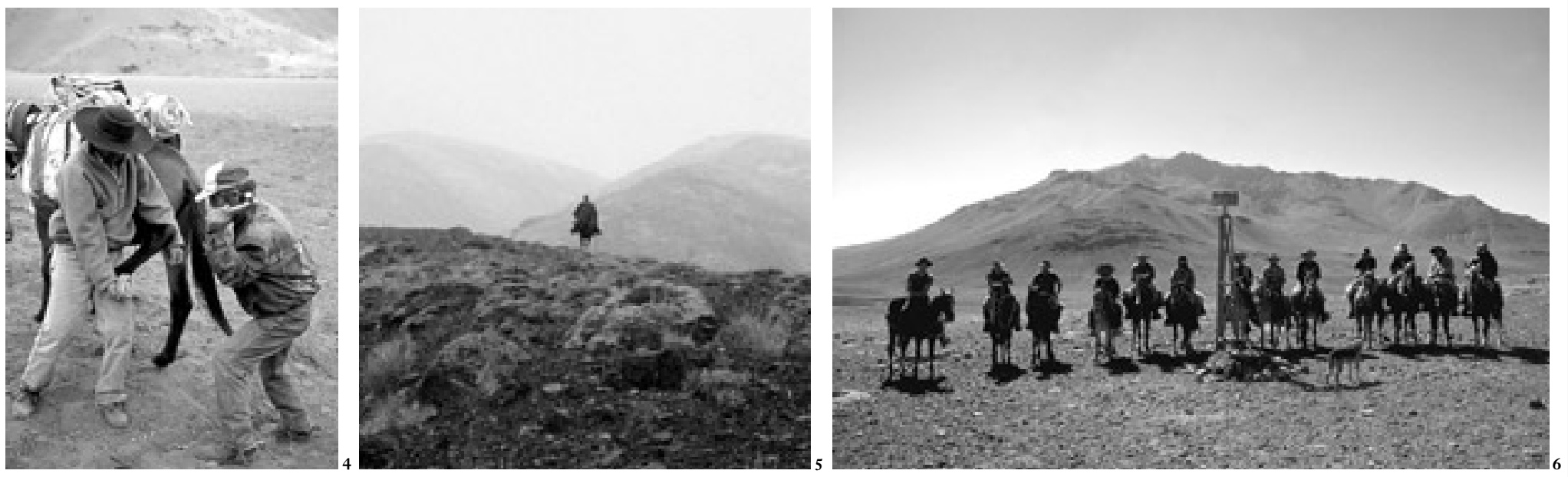


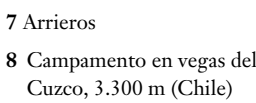

histórica de los Andes. De los 180 kilos de libros que adquirimos para el estudio del cruce de los Andes, encontramos sólo 25 páginas útiles, con información exacta de crónicas y mapas; lo mismo sucede con la incipiente información de botánica y geología alto andina.

No hay estética para la colonización de los Andes.

Ante esta necesidad surge sólido el gran tronco estético del Mediterráneo del sur, que trabaja sólo para descifrar el habla poética de un paisaje montañoso. Los fundadores conocidos de esta tradición son los poetas bíblicos del Génesis.

Estos maestros de la poesía hicieron ver a todo un pueblo que la tierra que habitaban hablaba: zarzas ardientes, truenos de las montañas, manantiales que brotan de las piedras, mares que se abren y se cierran, diluvios, etc.

Es tan fuerte esa poesía que llega a constituirse en la voz de un Dios único, que nunca hasta hoy hombre alguno ha visto, que ni siquiera se puede nombrar y que solamente habla a través del paisaje. "Háblenme montes y valles...".

Hace dos años estuve en la tierra prometida participando en un simposio de escultura.

Trabajé sus piedras, mármoles de las tierras de Canaán, lugar de las "Bodas de Canaán", esas rocas, que fueron la voz del mismo Dios. En esos secanos pedregosos entendí el poder de sus poetas fundadores de la poesía occidental.

Ni el valle más seco del Norte Chico es tan árido como Israel, y cualquier molle del río Elqui es más verde que cualquier cedro de los montes del Líbano, bajo cuya sombra trabajé. Fue, quizás, esa aridez que transportaron las páginas de la Biblia, la arena fundadora de la poesía de Gabriela Mistral.

La valentía de los poetas bíblicos está en Gabriela, también el entendimiento místico del paisaje y la materia. La precisión de vida o muerte en las palabras para poner el primer puente entre el chileno y su paisaje perdido:

\section{La montaña de noche}

"Me va ganando el corazón el frío

de la cumbre cercana. Pienso: acaso

los muertos que dejaron por impuras

las ciudades, elijan el regazo

recóndito de los desfiladeros

de tajo azul,(...)

Termino con el principio del poema que equivale al "No temas" de los Ángeles del Génesis.

"Haremos fuego sobre la montaña

...jHaremos 30 fuegos brilladores!"

Gabriela Mistral en Tala, nos presenta los Andes llenos de luz y deja encerrados en ellos un enigma poético que no logro descifrar.

\section{...Alta ciudad Torres doradas}

Pascual arribo de mi gente,

Arca tendida de la alianza.

Pascua viene de paso (Paso de los Andes), muerte y resurrección.

Sé que algo tiene que morir en Chile, para poder entrar en las Torres doradas de los Andes.

$\mathrm{Al}$ final de este escrito comienzo a entender que el problema no es el miedo a los muertos del cerro, ni a la soledad, sino de vivir rodeados de tanta belleza, sintiendo que no la merecemos.

Causado, quizá, por la larga la guerra de Arauco,
Consultores de proyecto

Adolfo Ibáñez: Historiado

Isabel Cruz: Historiadora

Miguel Laborde: Cronista

Alfredo Jadresic: Médico

Patricia Samsing. Poeta

Ignacio Silva: Geólogo

Douglas Hübner: Periodista-

editor del libro

osé Moreno: Fotógrafo

(diaporamas)
Pablo Orrego: Fotógrafo Rodolfo Gajardo: Botánico de alto andino

Federico Lübert: Botánico Marcial Taverne: Agrónomo guía de cordillera María Paz Vargas: Veterinaria Arrieros: Lado chileno, Hugo Arancibia

Lado Argentino, Ramón Ossa la tristeza que ella generó, sumado al hecho de ser durante siglos la última colonia del sur. Desde esa falta de autoestima nos gobierna ese Chamán oscuro, que ha logrado generar, finalmente, un país de la Bella y la Bestia.

Hay un hechizo que matar en el "Cruce de los Andes". El primero lo hace San Martín hace 200 años con 10.600 mulas.

El segundo gran viaje hacia los Andes se lo propone Gabriela a Chile, en "Cordillera": Un viaje donde Chile asuma la belleza de su paisaje andino.

\section{¡Suelde el caldo de tus metales}

los pueblos rotos de tus abras;

Puño de bielo, palma de fuego A bielo y fuego purifícanos!

Alta ciudad Torres doradas

Pascual arribo de tu gente,

Arca tendida de la Alianza.

Termino con Gabriela porque ella es campesina de montaña, por eso le creo, le creo porque los montañeses casi nunca levantan la voz.

Le creo porque estos pequeños versos finales de Cordillera son los únicos que cuadran en algo con las siete cumbres que me rodean mientras escribía, tratando de entenderlas.

Cerro Damas, San Ramón, Bandurrias, Coironal, Purgatorio, Los Azules y Pechuga de Tórtola.

Pirque, 15 de Abril 2001 ARQ
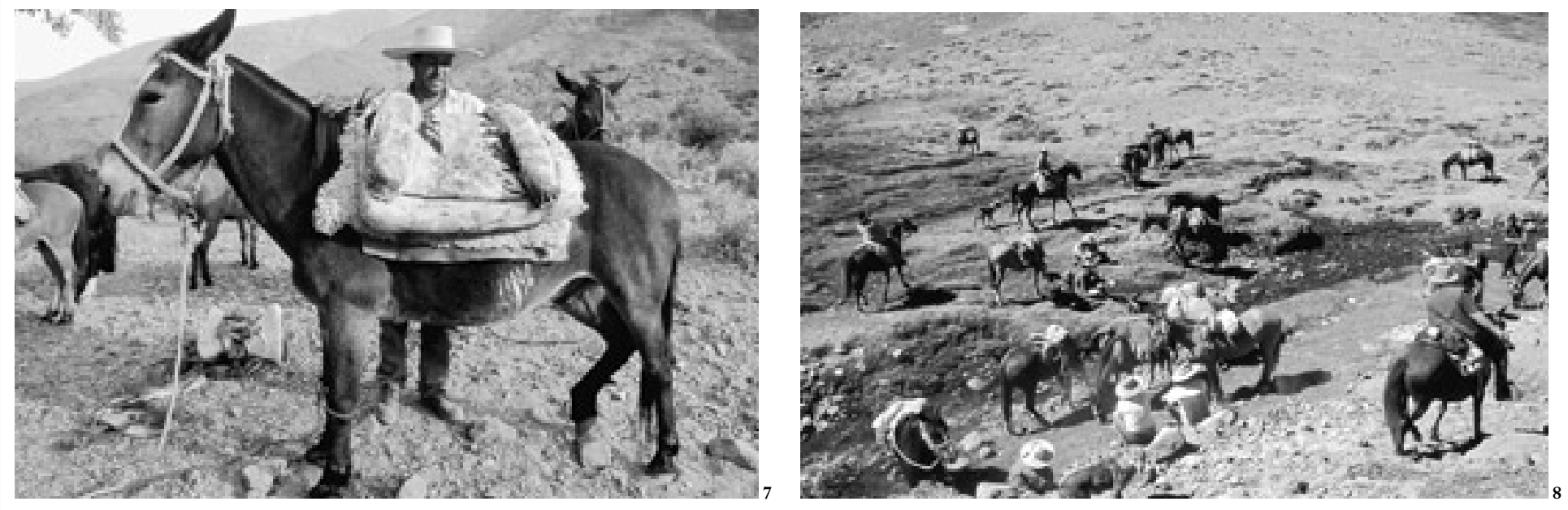